\title{
SERUM URIC ACID AND TARGET ORGAN DAMAGE IN ESSENTIAL HYPERTENSION AT MEDICINE DEPARTMENT OF GMC, BETTIAH, BIHAR
}

\begin{tabular}{|l|l|l|l|l|}
\hline Medicine & \\
\cline { 2 - 2 } & &
\end{tabular}

Dr. Mahendra M.B.B.S., M.D. (Med.),Senior Resident, Department ofMedicine, Govt. Medical College Kumar and MJK Hospital, Bettiah, W. Champaran, Bihar.

\section{Dr. Dharmendra}

Associate Professor, Department ofMedicine, Govt. Medical College and MJK Hospital, Prasad* Bettiah, W. Champaran, Bihar. *Corresponding Author

\begin{tabular}{ll}
\hline $\begin{array}{l}\text { Dr. } \\
\text { Parshuram Yugal }\end{array}$ & $\begin{array}{l}\text { Professor and Head of Department, Department ofMedicine, Govt. Medical College and } \\
\text { MJK Hospital, Bettiah, W. Champaran, Bihar. }\end{array}$ \\
\hline Dr. Debarshi Jana & $\begin{array}{l}\text { Young Scientist (DST) Institute of Post-Graduate Medical Education and Research, } \\
\text { A.J.C. Bose Road, Kolkata-700020, West Bengal, India. }\end{array}$ \\
\hline
\end{tabular}

\section{ABSTRACT}

Background: Hypertension is a major risk factor for cardiovascular mortality, as it acts through its effects on target organs, such as the heart and kidneys. Hyperuricemia increases cardiovascular risk in patients with hypertension.

Objective: To assess the relationship between serum uric acid and target organ damage (left ventricular hypertrophy and microalbuminuria) in untreated patients with essential hypertension.

Patients and methods: A cross-sectional study was carried out in 130 ( 85 females, 45 males) newly diagnosed, untreated patients with essential hypertension. Sixty-five healthy age- and sex-matched non-hypertensive individuals served as controls for comparison. Left ventricular hypertrophy was evaluated by cardiac ultrasound scan, and microalbuminuria was assessed in an early morning midstream urine sample by immunoturbidimetry. Blood samples were collected for assessing uric acid levels.

Results: Mean serum uric acid was significantly higher among the patients with hypertension $(379.7 \pm 109.2$ ìmol/L) than in the controls $(296.9 \pm 89.8 \mathrm{imol} / \mathrm{L} ; P<0.001)$, and the prevalence of hyperuricemia was $46.9 \%$ among the hypertensive patients and $16.9 \%$ among the controls $(P<0.001)$. Among the hypertensive patients, microalbuminuria was present in $54.1 \%$ of those with hyperuricemia and in $24.6 \%$ of those with normal uric acid levels $(P=0.001)$. Similarly, left ventricular hypertrophy was more common in the hypertensive patients with hyperuricemia ( $70.5 \%$ versus $42.0 \%$, respectively; $P=0.001$ ). There was a significant linear relationship between mean uric acid levels and the number of target organ damage (none versus one versus two: $P=0.012$ ).

Conclusion: These results indicate that serum uric acid is associated with target organ damage in patients with hypertension, even at the time of diagnosis; thus, it is a reliable marker of cardiovascular damage in our patient population.

\section{KEYWORDS}

essential hypertension, serum uric acid, left ventricular hypertrophy, microalbuminuria

\section{INTRODUCTION}

Hypertension is an important cardiovascular problem worldwide. Its prevalence is increasing in developing countries. Hypertension has adverse effects on various target organs, thus increasing the risk of stroke, coronary heart disease, and heart failure. This leads to high morbidity and mortality in many cases. Elevated blood pressure (BP) is also associated with an accelerated rate of decline in cognitive and renal function.

In evaluating a patient with hypertension, it is recommended that target organ damage (TOD) be identified in order to better define the individual's global cardiovascular risk in an effort to guide the decision to begin treatment, and to determine target BP levels. In less developed parts of the world, the diagnosis of hypertension is often delayed and, as a result, TOD may be present at the time of diagnosis. Uric acid (UA) levels tend to be elevated in patients with hypertension. Elevated UA is a risk factor for the development of cardiovascular disease, and the European Society of Hypertension-European Society of Cardiology guidelines recommend performing routine laboratory testing for serum UA (SUA) in patients with hypertension. While it has been shown that SUA is associated with multiple cardiovascular risk factors, including hypertension, metabolic syndrome, diabetes, and renal disease, it remains to be known if UA independently predicts adverse cardiovascular events in patients with hypertension. Various large trials have failed to identify UA as a significant and independent risk factor.

Identifying patients with subclinical TOD provides the best opportunity for preventing progression to overt cardiovascular disease. In clinical practice in limited-resource settings, it may not be practical to screen every newly diagnosed hypertensive individual for subclinical TOD due to limited access to facilities for echocardiography and microalbuminuria; therefore, identification of a relatively inexpensive risk marker, like UA, may help to identify patients who are at higher risk, thereby directing further evaluation.

\section{AIMS OF THE STUDY}

This study was thus aimed at investigating the relationship between SUA and left ventricular (LV) hypertrophy and microalbuminuria in newly diagnosed, untreated patients with essential hypertension.

\section{MATERIALAND METHODS}

Study design

This was a descriptive cross-sectional study carried out in the Department of Medicine, Govt. Medical College and MJK Hospital, Bettiah, W. Champaran, Bihar.

\section{Study population}

A total of 130 consecutive, never-treated, newly diagnosed hypertensive subjects aged between $30-70$ years of age were included in the study.

The subjects were characterized by: 1) average clinic systolic BP (SBP) values $>140 \mathrm{mmHg}$ or diastolic BP (DBP) $>90 \mathrm{mmHg}$, confirmed during two visits at the outpatient clinic; 2) no history or clinical evidence of congestive heart failure, myocardial infarction, arrhythmias, cardiac valve disease, coronary bypass surgery or angioplasty, diabetes mellitus and renal insufficiency, and no treatment with urate-lowering medication; and 3) no clinical evidence of secondary hypertension.

Allpatients returned to the ward after an overnight fast and underwent the following procedures: 1) clinic BP measurement; 2) venous blood sample collection for SUA; 3) spot midstream urine collection for microalbuminuria (patients with proteinuria on dipstick testing were excluded); 4) transthoracic echocardiogram; and 5) routine investigations (serum creatinine, fasting lipid profile, and plasma glucose).

Sixty-five non-hypertensive, healthy age- and sex-matched individuals were randomly selected from the hospital staff and patients' relatives and were classified as controls. 
Uric acid

UA was analyzed with the enzymatic colorimetric method using an autoanalyzer. Normal values in the hospital laboratory are $<360$ $\mu \mathrm{mol} / \mathrm{L}$ and $<420 \mu \mathrm{mol} / \mathrm{L}$ for women and men, respectively; therefore, individuals who had values above these levels were classified as having hyperuricemia.

\section{Fasting lipid profile}

Fasting cholesterol and triglyceride (TG) levels were measured using the enzymatic method with a reagent from Atlas Medical Laboratories (Atlas Development Corporation, Calabasas, CA, USA). Fasting highdensity lipoprotein (HDL) was measured with the precipitation method. Low-density lipoprotein (LDL) cholesterol values were calculated using the Friedewald equation when the TG level was $<4.0$ $\mathrm{mmol} / \mathrm{L}: \mathrm{LDL}=$ Total cholesterol $-(\mathrm{HDL}+\mathrm{TG} / 2.2)$

\section{Definition of an abnormal lipid profile:}

- $\quad$ Elevated $\mathrm{TG}=\mathrm{TG}>1.7 \mathrm{mmol} / \mathrm{L}$

- Hypercholesterolemia $=\mathrm{TC}>5.2 \mathrm{mmol} / \mathrm{L}$

- Low HDL cholesterol $=$ HDL cholesterol $<1.03 \mathrm{mmol} / \mathrm{L}$

- Elevated LDL cholesterol $=\mathrm{LDL}$ cholesterol $>3.0 \mathrm{mmol} / \mathrm{L}$.

\section{Microalbuminuria}

Randoximmunoturbidimetric assay for urinary albumin (Randox Laboratories, Antrim, UK) was used to determine microalbuminuria in an early morning spot midstream urine sample. Microalbuminuria was defined as an albumin concentration between $20-200 \mathrm{mg} / \mathrm{L}$. This is equivalent to albumin excretion of $30-300 \mathrm{mg} / 24$ hours (in a urine sample collected over 24 hours).

\section{Blood pressure measurements}

BP was measured with a standard mercury sphygmomanometer (with an appropriate cuff size) on the patients' right arm, as the patients were in the seated position with their feet on the floor after a 5-minute rest. SBP and DBP were taken at Korotkoff phases 1 and 5, respectively to the nearest $2 \mathrm{mmHg}$. The average of two BP measurements taken 5 minutes apart was used. The presence and severity of hypertension were then defined based on the seventh Joint National Committee on Prevention, Detection, Evaluation, and Treatment of High BP guidelines.

\section{Echocardiography}

Echocardiographic studies for all the patients were carried out with an AlokaProsound SSD 4000 echocardiography machine equipped with a $2.5 \mathrm{~Hz}$ transducer. With the patient in the left lateral decubitus position, targeted echocardiographic estimations were taken. These included the standard two-dimensional oriented motion-mode measurements of interventricularseptal thickness in diastole, LV posterior wall thickness in diastole, and LV end diastolic diameter just beyond the tips of the mitral valve leaflets. LV mass (in grams) was automatically calculated with the internal software of the machine.

LV mass was indexed to the body surface area using cut-off values of $134 \mathrm{~g} / \mathrm{m}^{2}$ and $110 \mathrm{~g} / \mathrm{m}^{2}$ for men and women, respectively.

Relative wall thickness (RWT) was calculated as $2 \times$ posterior wall thickness in end diastole/LV end diastolic diameter. A partition value of 0.45 for RWT was used for both men and women.

Patients with increased LV mass index (LVMI) and increased RWT were considered to have concentric hypertrophy, and those with increased LVMI and normal RWT were considered to have eccentric hypertrophy. Those with normal LVMI and increased or normal RWT were considered to have concentric remodeling or normal geometry, respectively.

\section{Definition of target organ damage}

TOD was defined by the presence of microalbuminuria (urinary albumin excretion: $20-200 \mathrm{mg} / \mathrm{L}$ ) or echocardiographic evidence of LV hypertrophy (LVH) defined as LVMI $\geq 134 \mathrm{~g} / \mathrm{m}^{2}$ in men and $\geq 110$ $\mathrm{g} / \mathrm{m}^{2}$ in women.

\section{Definition of degree of target organ damage}

The degree was determined by the number of target organs involved (ie, $0=$ no TOD; $1 \mathrm{TOD}=$ presence of either $\mathrm{LVH}$ or microalbuminuria; $2 \mathrm{TOD}=$ presence of both LVH and microalbuminuria).

\section{Statistical analysis}

All data were analyzed using the commercially available Statistical Package for the Social Sciences (SPSS) version 17.0 analytic software (IBM Corporation, Armonk, NY, USA). Data were expressed as mean \pm standard deviations, and frequencies as a percentage. Continuous variables were compared with the Students $t$-test, or one-way analysis of variance, as considered appropriate. Proportions or categorical parameters were compared with the chi-square test. Relations among continuous variables were assessed using Pearson's correlation coefficient and multiple linear regression analysis. All tests were considered to be statistically significant at the $P$-value $\leq 0.05$.

\section{RESULTS}

There were 130 patients with a female-to-male ratio of 1.9:1. The age range was $31-70$ years, with a mean age of $46.8 \pm 9.3$ years. The age range of the controls was $31-70$ years with a mean age of $44.4 \pm 9.8$ years. There was no statistically significant difference in the mean ages of the cases and controls $(P=0.094)$. The mean body mass index of the cases was $29.0 \pm 5.0 \mathrm{~kg} / \mathrm{m}^{2}$, which was significantly higher than that of the controls $\left(26.6 \pm 3.3 \mathrm{~kg} / \mathrm{m}^{2} ; P=0.001\right)$.

LVH was present in $55.4 \%$ of the cases and in $10.8 \%$ of the controls $(P<0.001)$, and the most common geometric pattern among the cases was concentric hypertrophy, while the majority of the controls had normal LV geometry. The prevalence of microalbuminuria among the cases was $37.7 \%$, while it was $7.7 \%$ in the controls $(P<0.001)$. Overall, $67 \%$ of the cases had at least one TOD, and females were more affected than males $(P=0.045)$.

The mean SUA was significantly higher in the cases $(379.7 \pm 109.2$ $\mu \mathrm{mol} / \mathrm{L})$ than in the controls $(296.9 \pm 89.8 \mu \mathrm{mol} / \mathrm{L} ; P<0.001)$, and the prevalence of hyperuricemia was $46.9 \%$ among the cases and $16.9 \%$ among the controls $(P<0.001)$. The mean SUA increased significantly with the severity of hypertension.

Microalbuminuria was present in $54.1 \%$ of the hypertensive cases with hyperuricemia, and in $24.6 \%$ of the cases with normal UA levels $(P=0.001)$. Similarly $\mathrm{LVH}$ was more common among the hypertensive cases with hyperuricemia compared to the hypertensive cases with normal serum uric acid levels $(70.5 \%$ versus $42.0 \%$ respectively, $P=0.001)$. Among the eleven $(16.9 \%)$ controls that had hyperuricemia, two had LVH and none had microalbuminuria.

SUA correlated positively with microalbuminuria among the cases ( $r=0.529 ; P<0.001)$, suggesting that in those with microalbuminuria, urine albumin excretion increased with increasing levels of SUA. This correlation remained significant even after controlling for the following variables: age $(r=0.535 ; P<0.001)$; body mass index (BMI) $(r=0.476 ; P=0.001)$; waist circumference (WC) $(r=0.489 ; P<0.001)$; SBP $(r=0.522 ; P<0.001)$; and DBP $(r=0.537 ; P<0.001)$, respectively. The correlation was not significant among the controls $(r=0.085 ; P=0.178)$. With multiple linear regression analysis, SUA was independently associated with microalbuminuria after adjusting for confounding variables including age, BMI, WC, SBP and DBP. The variables combined explained $31.1 \%$ of the variance observed $(F=3.154 ; P=0.012)$. SUA made a statistically significant and unique contribution to the variance in microalbuminuria $(\beta=0.517 ; P=0.001)$.

While the correlation between LVMI and SUA among the controls was not significant $(r=0.169 ; P=0.500)$, it was positive and significan among the cases $(r=0.336 ; P<0.001)$, with high levels of SUA associated with higher indexed LV mass. The correlation remained significant after controlling for age $(r=0.332 ; P<0.001)$; BMI $(r=0.303 ; P<0.001) ;$ WC $(r=0.287 ; P<0.001) ;$ SBP $(r=0.308$; $P<0.001)$; and DBP $(r=0.328 ; P<0.001)$. SUA levels were also independently associated with LVMI in regression analysis after adjusting for confounders including age, BMI, WC, SBP, and DBP. The variables combined explained $25.4 \%$ of the variance observed in LVMI $(P<0.001)$. SBP made the largest unique contribution $(P=0.001)$, although SUA also made a statistically significant contribution $(P=0.003)$.

There was a significant positive correlation between SUA level and the number of target organs involved $(r=0.255 ; P=0.017)$. This suggests that the number of TOD increased with increasing levels of SUA. Themean SUA level was higher in the cases with both microalbuminuria and LVH $(419.2 \pm 135.9 \mu \mathrm{mol} / \mathrm{L})$, than in those with either of these indices of TOD alone $(381.1 \pm 93.7 \mu \mathrm{mol} / \mathrm{L})$, or in those with none $(345.8 \pm 92.2 \mu \mathrm{mol} / \mathrm{L})$. This was statistically significant $(P=0.012)$ 


\section{CONCLUSION}

In conclusion, it is the opinion of the authors that in clinical practice in limited-resource settings, a cheap screening test like SUA should be done in all patients presenting with hypertension in order to identify those who are at higher total cardiovascular risk, and who thus warrant more intensive investigations and intervention.

\section{REFERENCES}

1. British Cardiac Society; British Hypertension Society; Diabetes UK; HEART UK; Primary Care Cardiovascular Society; Stroke Association JBS 2: Joint British Societies' guidelines on prevention of cardiovascular disease in clinical practice. Heart. 2005;91(Suppl 5):v1-v52.

2. Chobanian AV, Bakris GL, Black HR, et al. Joint National Committee on Prevention, Detection, Evaluation, and Treatment of High Blood Pressure National Heart, Lung, and Blood Institute; National High Blood Pressure Education Program Coordinating Committee. Seventh report of the Joint National Committee on Prevention, Detection, Committee. Seventh report of the Joint National Committee on Prevention, Detection,
Evaluation, and Treatment of High Blood Pressure. Hypertension. 2003;42 (6): Evaluation,

3. de Jong PE, Curhan GC. Screening, monitoring, and treatment of albuminuria: Public health perspectives. J Am SocNephrol. 2006;17(8):2120-2126.

4. EmokpaeAM, Abdu A. Serum uric acid levels among Nigerians with essential hypertension. Niger J Physiol Sci. 2013;28(1):41-44.

5. Poudel B, Yadav BK, Kumar A, Jha B, Raut KB. Serum uric acid level in newly diagnosed essential hypertension in a Nepalese population: a hospital based cross diagnosed essential hypertension in a Nepalese population:

6. Salako BL, Ogah OS, Adebiyi AA, et al. Unexpectedly high prevalence of target-organ Salako BL, Ogah OS, Adebiyi AA, et al. Unexpectedly high prevalence of target-organ
damage in newly diagnosed Nigerians with hypertension. Cardiovase J Afr. 2007;18(2):77-83

7. Whitworth JA, World Health Organization, International Society of Hypertension Writing Group 2003 World Health Organization (WHO)/ International Society of Hypertension (ISH) statement on management of hypertension. J Hypertens. 2003;21(11):1983-1992. 